\title{
Cosmological constant as a finite temperature effect
}

\author{
I. Y. Park \\ ${ }^{\dagger}$ Department of Applied Mathematics, Philander Smith College \\ Little Rock, AR 72223, USA \\ inyongpark05@gmail.com
}

\begin{abstract}
The cosmological constant problem is examined by taking an Einsteinscalar with a Higgs-type potential and scrutinizing the infrared structure induced by finite temperature effects. A variant optimal perturbation theory is implemented in the recently proposed quantumgravitational framework. The optimized renormalized mass, i.e., the renormalized mass determined by the variant optimal perturbation theory, of the scalar field turns out to be on the order of the temperature. This shifts the cosmological constant problem to compatibility of the consequent perturbative analysis. The compatibility is guaranteed essentially by renormalization group invariance of physical quantities. We point out the resummation behind the invariance.
\end{abstract}

Keywords: cosmological constant, finite temperature, quantum gravity 


\section{Introduction}

The cosmological constant (CC) problem [1] (see, e.g., [2-6] for reviews) arises from the loop effects of Standard Model (SM) particles, such as the Higgs particle. Since the electroweak scale is much higher than, say, the temperature of the cosmic microwave background (CMB), it is a standard practice to apply the zero-temperature setup to formulate and tackle the problem. However, since the $\mathrm{CC}$ as a vacuum energy is an infrared effect and thus governed by the low energy sector, the infrared structure of the theory must be important and its meticulous description is desirable. The fact that the $\mathrm{CC}$ is a vacuum energy also implies that quantization and renormalization of gravity must be involved in its systematic treatment. In particular, the solution of the problem would require renormalization of the vacuum energy. In this work we show that, when properly taken into account in the quantum gravitational setup, the finite temperature effects allow one to avoid the CC fine-tuning problem.

Are there indications that the finite temperature effects may a priori be important for $\mathrm{CC}$ analysis, despite the fact that the electroweak scale is much higher than the CMB temperature? First of all, it should definitely be possible, and is natural, to obtain zero-temperature results as a vanishingtemperature limit of the corresponding finite-temperature results. A hint of an indication comes from zero-temperature loop analysis, which typically yields logarithmic factors such as $\ln \frac{m}{\mu}$, where $m$ is the mass of the field and $\mu$ the renormalization scale. For the benefit of convergence, it is necessary to choose $\mu \sim m$. By the same token it will be necessary to take $\mu \sim m \sim T$ once the temperature enters. In the present work this scaling is achieved in the course of improving the perturbative analysis by optimal perturbation theory $(\mathrm{OPT})^{1}$ as well as standard thermal resummation: we show that there is an OPT procedure that enforces the scaling.

In the body we reformulate the $\mathrm{CC}$ problem as a zero-temperature limit of the finite temperature counterpart. A potential obstruction to any finitetemperature perturbative analysis is the well-known infrared problem. (Reviews can be found in [10-14].) For a high temperature, say, that of the QCD era, a well-known example is the 'Linde problem' [15], which has been an active topic of research; see [16] [17] and references therein. An effective field

\footnotetext{
${ }^{1} \mathrm{OPT}$ is based on the variational principle. Other thermal physics techniques based on the variational principle include screened perturbation theory [7] [8] and 2PI formalism [9].
} 
theory approach combined with lattice computation was proposed to deal with the problem [18]. The focus of the present work is a low temperature, the temperature of CMB. (Nevertheless, some of the textbook results obtained for a high temperature can be borrowed for reasons to be explained.) We show that the finite-temperature effects are in fact crucial: in particular, once the convergence property of the perturbation series is improved through a variant OPT [19], the optimized renormalized mass of the Higgs turns out to be essentially the temperature, ${ }^{2}$ thus removing the root of the CC fine-tuning problem.

With the renormalized mass determined, the following task still remains: the zero-temperature theories, such as the zero-temperature Standard Model, have been quite successful. There, the renormalized masses turn out to be close to the pole masses, usually within a few percent. In the case of the SM Higgs field, for instance, the renormalized mass is close to $125 \mathrm{GeV}$, the pole mass value. If one now wants to take the renormalized mass to be around the CMB temperature, which is much smaller than the pole mass, one must yet maintain compatibility with the zero-temperature analysis: the resulting perturbation theory should preserve the success of the original zerotemperature theory. In the body, after examining vast freedom in choosing renormalization conditions, we invoke renormalization group invariance of physical quantities to affirm this. We note that a certain resummation is behind the invariance.

With the renormalized mass being of the order of the temperature, the CC problem is avoided: the CMB temperature in terms of eV is $6.6 \times 10^{-4} \mathrm{eV}$. Approximating this as $10^{-12} \mathrm{GeV}$, the vacuum energy contribution associated with the thermal mass of a Higgs-type field is $\sim 10^{-48} \mathrm{GeV}^{4}$. This is roughly of the same order as the observed $\mathrm{CC}$ value $\sim 10^{-48} \mathrm{GeV}^{4}$.

\section{Freedom in subtraction scheme}

In section 3, we put forth an OPT that leads to the renormalized mass of the order of the temperature. This raises a question on consistency of the resulting perturbative analysis, since the new renormalized mass is much

\footnotetext{
${ }^{2}$ It has been revealed in the recent works of $[20,21]$ that quantum corrections can qualitatively change the classical solution. In the present work we see a similar novelty: the finite temperature non-perturbative effects dictate, small temperatures notwithstanding, the renormalized mass.
} 


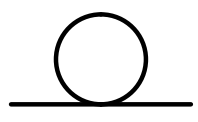

(a)

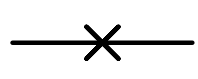

(b)

Figure 1: Diagrams for one-loop self-energy: (a) one-loop correction for propagator (b) its counter-term

smaller than the actual physical mass. Although in nature the modification amounts to finite renormalization and thus must not affect the physics, it will be useful to take a close look at how the perturbative analysis modifies in the new scheme. As we now demonstrate by considering two-loop renormalization of the propagator of a real scalar theory with a quartic potential, the key to preserving the success of the zero-temperature lies in vast freedom in choosing subtraction schemes.

Let us consider the following scalar system in a flat background

$$
S=-\int d^{4} x\left[\frac{1}{2} \partial_{\mu} \zeta \partial^{\mu} \zeta+\frac{1}{2} m^{2} \zeta^{2}\right]-\int d^{4} x \frac{\lambda}{4 !} \zeta^{4}
$$

The two-point proper vertex is defined as

$$
\Gamma^{(2)} \equiv k^{2}+m^{2}-\Sigma(k)
$$

where $\Sigma$ denotes self-energy. At one-loop, $\Sigma$ can be computed by considering the diagrams in Fig. 1: the one-loop two-point divergence introduces the counter-term:

$$
\longrightarrow \quad:-\frac{m^{2}}{4} \frac{\lambda}{(4 \pi)^{2}}\left(\frac{1}{\varepsilon}+c_{m}\right) \zeta^{2}
$$

where $\varepsilon \equiv \frac{4-D}{2}$ and $D$ denotes the spacetime dimension; $c_{m}$ is a constant to be determined by one's subtraction scheme. For instance, the modified minimal subtraction $(\overline{\mathrm{MS}})$ corresponds to setting $c_{m}=0$. Fixing the renormalized mass according to the OPT principle of minimal sensitivity (as we will in section 3 ) is in contrast to the usual practice in zero-temperature: there, one 

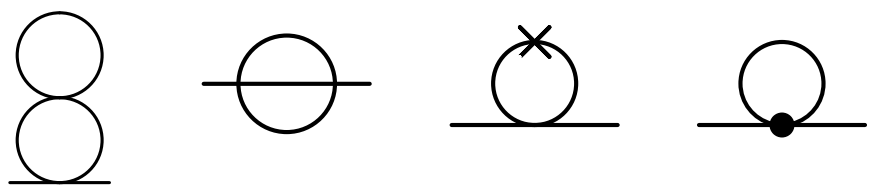

Figure 2: Diagrams for two-loop self-energy

fixes $c_{m}$ by one's subtraction scheme, and then the renormalized mass by the pole mass condition,

$$
k^{2}+m^{2}-\left.\Sigma(k)\right|_{k^{2}=-m_{P}^{2}}=0
$$

where $m_{P}$ denotes the physical pole mass. In the new scheme, it is the coefficient $c_{m}$ that is determined by the pole mass condition, while the renormalized mass is fixed by the OPT.

As stated in the introduction, the advantage of the new scheme is obvious: it realizes the scaling mentioned in the introduction and thereby allows one to avoid the $\mathrm{CC}$ fine-tuning problem. To see the disadvantage, let us note that the pole mass condition in the new scheme yields

$$
c_{m} m^{2} \sim m_{P}^{2}-m^{2}
$$

and thus implies a larger value of $c_{m}$, compared with the standard approach where the pole mass condition typically leads to $m^{2} \simeq m_{P}^{2}$. In general, for mass-related quantities it will not be possible at tree-level to achieve suitable agreement with experimental values, since the renormalized mass is pre-fixed: it will be necessary to go to one-loop where one has the freedom of adjusting the finite parts. The two-loop-relevant diagrams are given in Fig. 2. The circle in the last diagram in Fig. 2 represents the counter-term for the oneloop four-point diagram (not explicitly shown). Adding all up, the total two-loop self-energy $\Sigma^{(2)}$ is

$$
\Sigma^{(2)}=m^{2} \frac{\lambda^{2} \mu^{4 \varepsilon}}{(4 \pi)^{4}}\left[\frac{1}{2 \varepsilon^{2}}+\frac{1}{4 \varepsilon}\left(-1+c_{m}+3 c_{\lambda}\right)-\frac{p^{2}}{24 m^{2} \varepsilon}+\cdots\right]
$$

where $c_{\lambda}$ denotes the finite part of the counter-term of the one-loop four-point amplitude and $p$ the momentum entering through one end of the diagrams. 
The $\varepsilon$-pole terms will have to be removed by two-loop counter-terms. Let us focus on the finite parts. The constant $c_{m}$ will also appear in the finite parts represented by the ellipses. ${ }^{3}$ By imposing the pole mass condition and solving it, say, interactively for $c_{m}$, it will be possible to determine its twoloop correction. Since $\Sigma^{(2)}$ has an additional $\lambda$ and $\hbar$ compared with one-loop, the large value of $c_{m}$ cannot disturb the series in any significant way. After all, the new perturbation can be viewed as finite renormalization. We will point out later that a certain resummation is behind this finite renormalization.

\section{Variant OPT-induced renormalized mass}

The crux of the variant optimal perturbation theory (OPT) can be captured by considering a scalar system in a flat spacetime. Since the UV divergences originate locally from a short distance, they are insensitive to global geometry. For this reason, the zero-temperature UV regularization can be employed in a finite-temperature theory. As for quantities depending on the infrared structure, the prime example of which is vacuum energy, one must consider in principle the actual background. The difference between using the curved background and the flat one lies in the finite parts. (However, the finite parts are adjusted by the renormalization conditions anyway; we refer to [22] for further discussion.)

In thermal field theory, convergence of perturbative analysis is improved by resummation. The convergence can be further enhanced with a touch of non-perturbative techniques, such as OPT. The OPT implemented in this work is a relatively minor, but nonetheless crucial, variation of the widelystudied one. In the widely-used OPT, an artificial mass term is subtracted out after adding. This is one way of ensuring artificial-mass independence of the full closed results. In our case, the renormalized mass itself serves as the OPT parameter to be fixed by the OPT principle of minimal sensitivity.

\footnotetext{
${ }^{3}$ Since the issue under consideration belongs to the mass, one may well set $c_{\lambda}=0$ in the spirit of the $\overline{\mathrm{MS}}$. Similarly, the two-loop analogues of $c_{m}, c_{\lambda}$ (and the finite part associated with the wavefunction renormalization) can be set to zero. In other words, too much freedom in choosing the finite parts can be a burden: the freedom remaining after determining $c_{m}$ can be fixed just as in a convenient subtraction scheme, such as the $\overline{\mathrm{MS}}$, to facilitate next steps, e.g., solving the renormalization group equations.
} 
The gravity-scalar system that we consider is

$$
S=\frac{1}{\kappa^{2}} \int d^{4} x \sqrt{-g} R-\int d^{4} x \sqrt{-g}\left(\frac{1}{2} g^{\mu \nu} \partial_{\mu} \zeta \partial_{\nu} \zeta+V(\zeta)\right)
$$

where $\kappa^{2}=16 \pi G$ with $G$ being Newton's constant. The potential $V(\zeta)$ is

$$
V(\zeta)=\frac{\lambda}{4 !}\left(\zeta^{2}+\frac{6}{\lambda} \nu^{2}\right)^{2}
$$

Note the notation change as compared with section 2: the mass has been denoted by $\nu$. One conceptual hurdle is the justification of the complete-square form of the potential instead of the usual $V=\frac{1}{2} \nu^{2} \zeta^{2}+\frac{1}{4 !} \lambda \zeta^{4}$. The value of $\mathrm{CC}$ depends, of course, on whether one uses the complete-square form or the form without the constant piece. A shift of potential by a constant is immaterial in flat spacetime quantum field theory. The same is not true, however, in the quantum gravitational context. Whether one should use the completesquare form or the more usual form is not part of the CC problem. A closely related question, whose answer is not currently known [23], is why the minimum value of the classical Higgs potential should be taken to be zero. It is an independent problem that must ultimately be answered experimentally. Our goal here is to show that in the setup dictated largely by convergence of thermal perturbation theory, the fine tuning-problem is not present; this goal can be achieved more conveniently with the complete-square form.

To set the stage for the refined BFM $[22,24-26]$, we shift the fields as

$$
g_{\mu \nu} \rightarrow h_{\mu \nu}+\tilde{g}_{\mu \nu} \quad, \quad \zeta \rightarrow \hat{\zeta}+\tilde{\zeta}
$$

with

$$
\tilde{g}_{\mu \nu} \equiv g_{c \mu \nu}+\varphi_{\mu \nu} \quad, \quad \tilde{\zeta} \equiv \zeta_{c}+\xi
$$

where $g_{c \mu \nu}, \zeta_{c}$ denote the classical solutions, $\varphi_{\mu \nu}, \xi$ the background fields, and $h_{\mu \nu}, \hat{\zeta}$ the fluctuation fields. The loop analysis is based on the following two-point functions (see [26] for the conventions). For the metric,

$$
<h_{\mu \nu}\left(x_{1}\right) h_{\rho \sigma}\left(x_{2}\right)>=\tilde{P}_{\mu \nu \rho \sigma} \tilde{\Delta}\left(x_{1}-x_{2}\right)
$$

where the tensor $\tilde{P}_{\mu \nu \rho \sigma}$ is given by

$$
\tilde{P}_{\mu \nu \rho \sigma} \equiv \frac{\bar{\kappa}^{2}}{2}\left(\tilde{g}_{\mu \rho} \tilde{g}_{\nu \sigma}+\tilde{g}_{\mu \sigma} \tilde{g}_{\nu \rho}-\frac{1}{2} \tilde{g}_{\mu \nu} \tilde{g}_{\rho \sigma}\right) ;
$$


where $\bar{\kappa}^{2} \equiv 2 \kappa^{2}$ and satisfies

$$
\tilde{P}_{\mu \nu \kappa_{1} \kappa_{2}} \tilde{P}_{\rho \sigma}^{\kappa_{1} \kappa_{2}}=\tilde{P}_{\mu \nu \rho \sigma}
$$

$\tilde{\Delta}\left(x_{1}-x_{2}\right)$ is Green's function for a (massless) scalar theory in the background metric $\tilde{g}_{\mu \nu}$ :

$$
<\hat{\zeta}\left(x_{1}\right) \hat{\zeta}\left(x_{2}\right)>=\tilde{\Delta}\left(x_{1}-x_{2}\right)
$$

The explicit form of $\tilde{\Delta}$ for a massive scalar theory will be given and utilized in section 3.2 where the curved space analysis of the matter-involving sector is conducted.

\subsection{Flat spacetime analysis}

As stated in the beginning, the crux of our OPT is captured by considering a scalar system in a flat spacetime. We employ the $\overline{\mathrm{MS}}$ subtraction scheme in the present section. We will come back to the deviation from the $\overline{\mathrm{MS}}$ scheme in section 3.3.

The starting point of the OPT-improved thermal resummation can be taken as the following renormalized action

$$
S(\zeta)=-\int d^{4} x \frac{1}{2} \partial_{\mu} \zeta \partial^{\mu} \zeta-\int d^{4} x\left(\frac{1}{2} M^{2} \zeta^{2}+\frac{\lambda}{4 !} \zeta^{4}\right)-\int d^{4} x \frac{3 \nu^{4}}{2 \lambda}
$$

with

$$
M^{2}(T) \equiv \nu^{2}+\frac{\lambda}{24} T^{2}
$$

Shift the field

$$
\zeta \rightarrow \hat{\zeta}+\tilde{\zeta}
$$

where $\hat{\zeta}, \tilde{\zeta}$ denote the fluctuation field and background field, respectively. Since we are interested in the potential as opposed to the action, the background field $\tilde{\zeta}$ can be treated as a constant. Then the potential can be effectively computed by considering the field-dependent mass term

$$
M^{2}(T) \rightarrow \tilde{M}^{2}(T, \tilde{\zeta})=\nu^{2}+\frac{\lambda}{24} T^{2}+\frac{\lambda}{2} \tilde{\zeta}^{2}
$$


and integrating out the fluctuation field $\hat{\zeta}$. A remark is in order before proceeding to determination of the OPT-induced renormalized mass. We stated earlier that although we consider a CMB-order temperature, the highenergy expansion can be utilized. The temperature being high or low is relative to the mass and we will show below that our OPT implies $\tilde{M} \sim \hbar^{1 / 2} T$ ( $\hbar$ will be kept implicit). One can now tell why the high-energy expansion is justified: since the auxiliary mass $\tilde{M}$ satisfies $\tilde{M} / T \sim \hbar^{1 / 2}$, the intermediate analysis corresponds to that of high temperature: $\frac{\tilde{M}}{T}<<1$.

The two-loop calculation of the effective potential was conducted long ago, e.g., in [27], [28], and [29]. For our goal, it is necessary to keep tract of the field-independent terms as well. Also, the $M$ - and $\tilde{M}$-dependence is important. Let us focus on the one-loop potential; after carefully following these terms, one gets

$$
\begin{aligned}
& V_{\mathrm{opt}}(\tilde{\zeta})=\frac{3 \nu^{4}}{2 \lambda}-\frac{\pi^{2} T^{4}}{90}-\frac{\tilde{M}^{4}}{32 \pi^{2}} \ln \frac{\bar{\mu} e^{\gamma_{E}}}{4 \pi T}+\frac{1}{24} \tilde{M}^{2} T^{2} \\
& +\frac{1}{2}\left(\nu^{2}+\frac{\lambda}{24} T^{2}\right) \tilde{\zeta}^{2}-\frac{1}{12 \pi} \tilde{M}^{3} T+\frac{1}{4 !} \lambda \tilde{\zeta}^{4}+\mathcal{O}\left(\frac{\tilde{M}^{6}}{T^{2}}\right)
\end{aligned}
$$

where $\bar{\mu} \equiv \mu\left(\frac{4 \pi}{e^{\gamma} E}\right)^{1 / 2}$ is a scaling parameter of dimensional regularization (with the $\overline{\mathrm{MS}}$ scheme). The field equation associated with (22), $\frac{\partial}{\partial \tilde{\zeta}} V_{\mathrm{opt}}=0$, yields

$$
\frac{\lambda}{6} \tilde{\zeta}^{2}+M^{2}-\frac{\lambda}{(4 \pi)^{2}} \tilde{M}^{2} \ln \frac{\bar{\mu} e^{\gamma_{E}}}{4 \pi T}+\frac{1}{24} \lambda T^{2}-\frac{1}{12 \pi} \tilde{M}^{3} T=0
$$

up to terms of two-loop order. The solution is

$$
\tilde{\zeta}^{2}(M) \simeq-\frac{6 M^{2}}{L}+\frac{1}{4 \pi^{2}}\left(-\pi^{2} T^{2}+3 \sqrt{2} \pi T \sqrt{-M^{2}}-3 M^{2} \ln \frac{\bar{\mu} e^{\gamma_{E}}}{4 \pi T}\right) .
$$

With this, one gets the following onshell potential:

$$
\begin{gathered}
V_{\mathrm{opt}}=\frac{3 \nu^{4}}{2 \lambda}-\frac{\pi^{2} T^{4}}{90}-\frac{\tilde{M}^{4}}{32 \pi^{2}} \ln \frac{\bar{\mu} e^{\gamma_{E}}}{4 \pi T}+\frac{1}{24} \tilde{M}^{2} T^{2} \\
+\frac{1}{2} M^{2} \tilde{\zeta}^{2}(M)-\frac{1}{12 \pi} \tilde{M}^{3} T+\frac{1}{4 !} \lambda \tilde{\zeta}^{4}(M)+\mathcal{O}\left(\frac{\tilde{M}^{6}}{T^{2}}\right) .
\end{gathered}
$$

By solving the following PMS condition for $\nu^{2}$

$$
\frac{\partial V_{\mathrm{opt}}}{\partial \nu^{2}}=0
$$


one gets ${ }^{4}$

$$
\nu^{2}=-\frac{5}{72} \lambda T^{2}
$$

which leads to

$$
\zeta^{2}=-\frac{T^{2}}{12}
$$

Once the potential (22) is evaluated with this value, one gets

$$
V_{\text {opt }}=-\frac{\pi^{2}}{90} T^{4} \sim T^{4}
$$

which then allows one to avoid the fine-tuning problem.

The complete two-loop offshell form of the potential will be presented in [30]. There one encounters a novelty: the potential develops an imaginary part, signaling instability of the vacuum. ${ }^{5}$ More on this in the conclusion.

\subsection{Curved space analysis}

Whereas what we referred to as the second-layer perturbation in [22] is necessary for the pure gravity sector computation, there exists, for the matter sector, a powerful shortcut based on the first-layer perturbation, the "onestroke" method. In the present work the first-layer perturbation will be used exclusively. From the results obtained, it becomes evident that the qualitative conclusion of the flat space analysis remains unchanged.

\section{Graviton sector}

Let us recall the zero-temperature case first. In [25] and [22], we conducted the computation in a brute-force manner by employing the second-layer perturbation and viewing the classical CC as the graviton mass term. As shown,

\footnotetext{
${ }^{4} \mathrm{~A}$ similar result of $\nu^{2} \sim \lambda T^{2}$ was obtained in [31] (see also [32] and [33]) by considering renormalization group and choosing appropriate renormalization conditions. More on this in section 3.3.

${ }^{5}$ Strictly speaking, the potential itself remains real even at two-loop. However, this is because the source of the imaginary parts, $\tilde{M}^{3}$, does not contribute even at two-loop. Due to the expected contribution of $\tilde{M}^{3}$ term (and the terms with higher odd-integer powers of $\tilde{M}$ that should appear in higher-loop computations), it is expected that the complexity of the potential will become manifest at three-loop and on.
} 
e.g., in [22], the result is a divergent $\mathrm{CC}$ term

$$
\sim \int \sqrt{-\tilde{g}}
$$

Strictly speaking, for a flat background, the one-loop results vanish in dimensional regularization in the absence of the classical CC treated as the graviton mass term. For consistency with the observed value of the $\mathrm{CC}$, the classical (i.e., renormalized) $\mathrm{CC}$ will have to be set to $\sim T^{4}$ in the renormalization program described below, and thus will not qualitatively affect the proposed resolution of the CC problem.

The results of $n$-loop with $n \geq 2$ vanish, irrespective of the finite temperature contribution, due to the tracelessness of $P_{\mu \nu \rho \sigma}$ and (13). An arbitrary $n$-loop graph with $n \geq 2$ contains a product of vertices that can be written as

$$
<h_{\alpha_{1} \alpha_{2}} h_{\alpha_{3} \alpha_{4}} h_{\alpha_{5} \alpha_{6}} \cdots h^{\beta_{1} \beta_{2}} h^{\beta_{3} \beta_{4}} h^{\beta_{5} \beta_{6}} \cdots>
$$

where the upper and lower indices are fully contracted. Contractions of the fields lead to

$$
\tilde{P}_{\mu_{1} \nu_{1} \rho_{1} \sigma_{1}} \tilde{P}_{\mu_{2} \nu_{2} \rho_{2} \sigma_{2}} \cdots
$$

where again, all the indices are contracted one way or another with $\tilde{g}^{\mu \nu}$ 's. Whenever a pair of $\tilde{P}^{\prime}$ 's have a pair of the indices contracted, the explicit expression for $\tilde{P}$ given in (12) can be used. One can also use (13) to reduce the total number of $\tilde{P}^{\prime}$ s until only one $\tilde{P}$ remains at the end. Since all of the indices must be contracted, the final expression must be $\sim \tilde{P}_{\mu \nu}{ }^{\mu \nu}$, and therefore vanishes. One important implication of this analysis is that it is not necessary to conduct resummation or OPT in the gravity sector.

\section{Matter-involving sector}

The matter-sector diagrams can be subdivided, depending on whether or not they involve a graviton loop. The matter-involving vertices do not, unlike the pure graviton vertices, come with $\frac{1}{\kappa^{2}}$. Since the graviton propagator comes with $\kappa^{2}$, the diagrams leading in $\kappa$ are those with a matter loop, which are our focus.

There exists a highly effective "one-stroke" method of computing the matterinvolving part of the effective action, in which the flat spacetime analysis can 


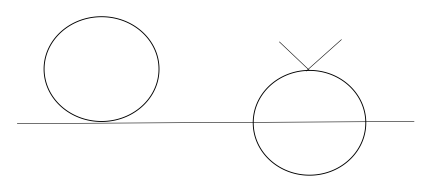

(a)

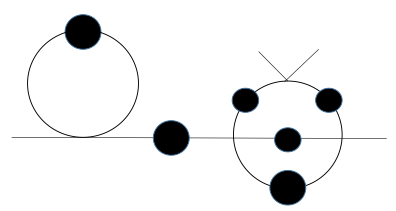

(b)

Figure 3: Resummation behind the new scheme: (a) a connected diagram in $\overline{\mathrm{MS}}$ scheme (b) resummation required in the new scheme

be entirely carried over. For this, note the explicit form of $\tilde{\Delta}\left(x_{1}-x_{2}\right)$ can be written as

$$
\tilde{\Delta}\left(x_{1}-x_{2}\right)=\int \frac{d^{4} k}{(2 \pi)^{4}} \frac{1}{\sqrt{-\tilde{g}\left(x_{1}\right)}} \frac{e^{i k \cdot\left(x_{1}-x_{2}\right)}}{i k_{\mu} k_{\nu} \tilde{g}^{\mu \nu}\left(x_{1}\right)} .
$$

Defining "flattened" momentum and coordinates as

$$
K_{\underline{\alpha}} \equiv \tilde{e}_{\underline{\alpha}}^{\mu} k_{\mu} \quad, \quad X \underline{\beta} \equiv \tilde{e}_{\bar{\nu}}^{\underline{\beta}} x^{\nu} \quad, \quad \tilde{e}_{\underline{\alpha}}^{\mu} \tilde{e}_{\underline{\beta}}^{\nu} \tilde{g}_{\mu \nu}=\eta_{\underline{\alpha} \beta}
$$

where the underlined indices are flattened, one gets the flattened propagator:

$$
\tilde{\Delta}\left(X_{1}-X_{2}\right)=\int \frac{d^{4} K}{(2 \pi)^{4}} \frac{e^{i K_{\underline{\gamma}}\left(X_{1}-X_{2}\right)^{\underline{\underline{q}}}}}{i K_{\underline{\alpha}} K_{\underline{\beta}} \eta^{\underline{\alpha} \underline{\beta}}} .
$$

When computing a diagram, one can pull out all of the background fields and contract the fluctuation fields. The propagators can then be transformed to the above. Afterward, the steps become parallel to those corresponding to the flat cases. The matter part of the effective action can thus be computed exactly in the same manner in which it is computed in the flat case.

\subsection{Consistency of new subtraction scheme}

With the renormalized mass around the CMB temperature, one should make sure that that framework preserves the success of the zero-temperature theory such as that seen in zero-temperature SM. The new perturbation has been 
illustrated in section 2 by taking a simple scalar theory. In the case of the SM, each sector in the SM, i.e., the Higgs, gauge, and fermion, the renormalization process should be modified from that of the standard renormalization scheme, e.g., $\overline{\mathrm{MS}}$. More specifically, in the modified scheme, the pole mass value must be realized by adjusting the finite parts of the divergent integrals that are chosen differently from those in the standard renormalization scheme: in analysis with $\overline{\mathrm{MS}}$, the pole mass condition is part of the $\overline{\mathrm{MS}}$ scheme, and the renormalized mass is not determined prior to the pole mass condition. It is the pole mass condition that determines the renormalized mass. In contrast, in the new scheme the renormalized mass is determined, as demonstrated in section 3.1, by the OPT. The finite parts are to be determined by the physical pole mass condition. What it implies is that it is necessary to go to the oneloop level to achieve the accuracy of amplitudes obtained in $\overline{\mathrm{MS}}$. In order to match the values of, e.g., the tree amplitudes computed in $\overline{\mathrm{MS}}$, one must insert one-particle-irreducible diagrams to the internal lines. (We anticipated that one-loop insertion will normally be sufficient.) The insertions amount to a certain resummation. The situation is generally illustrated in Fig. 3. In light of this, it is also worth noting that the $\nu^{2} \sim T^{2}$ scaling was previously obtained in [31] by choosing appropriate renormalization conditions.

\section{Conclusion}

Since some of the Standard Model particles, such as the Higgs, are massive, the matter contributions to the $\mathrm{CC}$ are naively expected to be larger than that of the graviton. The variant OPT reveals, however, that the ultimate determining factor of the $\mathrm{CC}$ is the temperature. We believe that this identifies the cosmological constant problem at its root. This leads to necessity of employing a new renormalization scheme. In the new scheme, one needs to go a few orders higher and perform a certain resummation to achieve the same level of proximity to the values of the physical observables as in the zero-temperature standard schemes. Given what it brings, this seems to a relatively small price to pay.

We end with three ramifications of our results and future directions. The results obtained in this work suggest that, earlier in the thermal history of the Universe, the value of the $\mathrm{CC}$ should have been larger. In other words the $\mathrm{CC}$ becomes time-dependent through the temperature, and the present small value must be due to the age of the Universe [23]. More quantitatively, 
the following is the basis for this anticipation. In [25] it was shown that there exists a time-dependent solution that approaches the minimal value of the potential. By using the one-parameter family of the potentials labeled by the temperature, one can repeat the analysis with the present finite temperature potential. One can then analyze the resulting quantum-level action, and it should be possible, at the time-dependent solution level, to establish a CC that decreases to a small value. One may introduce a renormalized CC, $\Lambda_{\text {ren }}$. To be consistent with the fact that the observed value of the CC is small, one will have to take $\Lambda_{\text {ren }}$ to be small. It will be of interest to pursue this line of study. Another ramification, not unrelated to the first, is that the large value of the CC becomes natural when the temperature is on the order of the EW scale. (This may be something profound, and have implications for the hierarchy problem in the SM.) Since the Universe was at higher temperatures in the previous eras, it will be a meaningful endeavor to explore whether one could come up with a streamlined description covering the entire temperature range, say, from the near-Planck era to the present. With the recent progress in the OPT literature, the present results indicate toward an affirmative answer. Lastly, as we will report in [30], the potential is expected to develop a imaginary part. We interpret this as an indication of the vacuum decay from a finite temperature to zero temperature. Just as an analogous decay induced by a bounce worm hole solution plays an important role in black hole information [34] [35], the present vacuum is likely to have interesting cosmological implications that deserve further study. 


\section{References}

[1] S. Weinberg, "The Cosmological Constant Problem," Rev. Mod. Phys. 61, 1-23 (1989) doi:10.1103/RevModPhys.61.1

[2] T. Padmanabhan, "Cosmological constant: The Weight of the vacuum," Phys. Rept. 380, 235-320 (2003) doi:10.1016/S0370-1573(03)00120-0 [arXiv:hep-th/0212290 [hep-th]].

[3] E. Bianchi and C. Rovelli, "Why all these prejudices against a constant?," [arXiv:1002.3966 [astro-ph.CO]].

[4] J. Martin, "Everything You Always Wanted To Know About The Cosmological Constant Problem (But Were Afraid To Ask)," Comptes Rendus Physique 13, 566-665 (2012) doi:10.1016/j.crhy.2012.04.008 [arXiv:1205.3365 [astro-ph.CO]].

[5] J. Sola, "Cosmological constant and vacuum energy: old and new ideas," J. Phys. Conf. Ser. 453, 012015 (2013) doi:10.1088/17426596/453/1/012015 [arXiv:1306.1527 [gr-qc]].

[6] C. Moreno-Pulido and J. Sola, "Running vacuum in quantum field theory in curved spacetime: renormalizing $\rho_{v a c}$ without $\sim m^{4}$ terms," Eur. Phys. J. C 80, no.8, 692 (2020) doi:10.1140/epjc/s10052-020-8238-6 [arXiv:2005.03164 [gr-qc]].

[7] F. Karsch, A. Patkos and P. Petreczky, "Screened perturbation theory," Phys. Lett. B 401, 69-73 (1997) doi:10.1016/S0370-2693(97)003924 [arXiv:hep-ph/9702376 [hep-ph]].

[8] J. O. Andersen and M. Strickland, "Mass expansions of screened perturbation theory," Phys. Rev. D 64, 105012 (2001) doi:10.1103/PhysRevD.64.105012 [arXiv:hep-ph/0105214 [hep-ph]].

[9] J. P. Blaizot, E. Iancu and A. Rebhan, "Approximately selfconsistent resummations for the thermodynamics of the quark gluon plasma. 1. Entropy and density," Phys. Rev. D 63, 065003 (2001) doi:10.1103/PhysRevD.63.065003 [arXiv:hep-ph/0005003 [hep-ph]].

[10] J. I. Kapusta and C. Gale, "Finite-Temperature Field Theory: Principles and Applications," Cambridge university press (2006) 
[11] M. Le Bellac, "Thermal Field Theory," Cambridge university press (2000)

[12] J. P. Blaizot, "Quantum Fields at Finite Temperature "from tera to nano Kelvin"," Soryushiron Kenkyu Electron. 119, 11-108 (2011) doi:10.24532/soken.119.2_11 [arXiv:1108.3482 [hep-ph]].

[13] M. Laine and A. Vuorinen, "Basics of Thermal Field Theory," Lect. Notes Phys. 925, pp.1-281 (2016) doi:10.1007/978-3-319-31933-9 [arXiv:1701.01554 [hep-ph]].

[14] E. Senaha, "Symmetry Restoration and Breaking at Finite Temperature: An Introductory Review," Symmetry 12, no.5, 733 (2020) doi:10.3390/sym12050733

[15] A. D. Linde, "Infrared Problem in Thermodynamics of the YangMills Gas," Phys. Lett. B 96, 289-292 (1980) doi:10.1016/03702693(80)90769-8

[16] J. Ghiglieri, A. Kurkela, M. Strickland and A. Vuorinen, "Perturbative Thermal QCD: Formalism and Applications," Phys. Rept. 880, 1-73 (2020) doi:10.1016/j.physrep.2020.07.004 [arXiv:2002.10188 [hep-ph]].

[17] Q. Du, M. Strickland, U. Tantary and B. W. Zhang, "Two-loop HTL-resummed thermodynamics for $\mathcal{N}=4$ supersymmetric YangMills theory," JHEP 09, 038 (2020) doi:10.1007/JHEP09(2020)038 [arXiv:2006.02617 [hep-ph]].

[18] E. Braaten and A. Nieto, "Effective field theory approach to high temperature thermodynamics," Phys. Rev. D 51, 6990-7006 (1995) doi:10.1103/PhysRevD.51.6990 [arXiv:hep-ph/9501375 [hep-ph]].

[19] P. M. Stevenson, "Optimized Perturbation Theory," Phys. Rev. D 23, 2916 (1981) doi:10.1103/PhysRevD.23.2916

[20] A. J. Nurmagambetov and I. Y. Park, "Quantum-induced trans-Planckian energy near horizon," JHEP 05, 167 (2018) doi:10.1007/JHEP05(2018)167 [arXiv:1804.02314 [hep-th]].

[21] A. J. Nurmagambetov and I. Y. Park, "Quantum-gravitational transPlanckian radiation by a rotating black hole," [arXiv:2007.06070 [hepth]]. 
[22] I. Y. Park, "Revisit of renormalization of Einstein-Maxwell theory at one-loop," [arXiv:1807.11595 [hep-th]], to appear in PTEP (ptaa167, https://doi.org/10.1093/ptep/ptaa167).

[23] S. Weinberg, "Cosmology," Oxford university press (2008)

[24] I. Y. Park, "Lagrangian constraints and renormalization of 4D gravity," JHEP 04, 053 (2015) doi:10.1007/JHEP04(2015)053 [arXiv:1412.1528 [hep-th]].

[25] I. Y. Park, "One-loop renormalization of a gravity-scalar system," Eur. Phys. J. C 77, no. 5, 337 (2017) doi:10.1140/epjc/s10052-017-4896-4 [arXiv:1606.08384 [hep-th]].

[26] I. Park, "Foliation-Based Approach to Quantum Gravity and Applications to Astrophysics," Universe 5, no.3, 71 (2019) doi:10.3390/universe5030071 [arXiv:1902.03332 [hep-th]].

[27] R. R. Parwani, "Resummation in a hot scalar field theory," Phys. Rev. D 45, 4695 (1992) doi:10.1103/PhysRevD.45.4695 [arXiv:hep-ph/9204216 [hep-ph]].

[28] P. B. Arnold and O. Espinosa, "The Effective potential and first order phase transitions: Beyond leading-order," Phys. Rev. D 47, 3546 (1993) doi:10.1103/PhysRevD.47.3546 [arXiv:hep-ph/9212235 [hep-ph]].

[29] S. Chiku and T. Hatsuda, "Optimized perturbation theory at finite temperature," Phys. Rev. D 58, 076001 (1998) doi:10.1103/PhysRevD.58.076001 [arXiv:hep-ph/9803226 [hep-ph]].

[30] I. Y. Park, "Quantization of gravity and finite temperature effects," Manuscript in preparation

[31] J. P. Blaizot and N. Wschebor, "Massive renormalization scheme and perturbation theory at finite temperature," Phys. Lett. B 741, 310-315 (2015) doi:10.1016/j.physletb.2014.12.040 [arXiv:1409.4795 [hep-ph]].

[32] J. L. Kneur and M. B. Pinto, "Scale Invariant Resummed Perturbation at Finite Temperatures," Phys. Rev. Lett. 116, no.3, 031601 (2016) doi:10.1103/PhysRevLett.116.031601 [arXiv:1507.03508 [hep-ph]]. 
[33] J. L. Kneur and M. B. Pinto, "Renormalization Group Optimized Perturbation Theory at Finite Temperatures," Phys. Rev. D 92, no.11, 116008 (2015) doi:10.1103/PhysRevD.92.116008 [arXiv:1508.02610 [hep-ph]].

[34] I. Y. Park, "Foliation-based quantization and black hole information," Class. Quant. Grav. 34, no.24, 245005 (2017) doi:10.1088/13616382/aa9602 [arXiv:1707.04803 [hep-th]].

[35] I. Y. Park, "Black hole evolution in quantum-gravitational framework," [arXiv:1912.07413 [hep-th]]. 